\title{
Approach to asymptotically diffusive behavior for Brownian particles in periodic potentials : extracting information from transients.
}

\author{
David S. Dean ${ }^{1}$ and Gleb Oshanin ${ }^{2,3}$ \\ ${ }^{1}$ Université de Bordeaux and CNRS, Laboratoire Ondes et Matière d'Aquitaine (LOMA), UMR 5798, F-33400 Talence, France \\ ${ }^{2}$ Sorbonne Universités, UPMC Univ Paris 06, UMR 7600, LPTMC, F-75005, Paris, France \\ ${ }^{3}$ CNRS, UMR 7600, Laboratoire de Physique Théorique de la Matière Condensée, F-75005, Paris, France
}

\begin{abstract}
A Langevin process diffusing in a periodic potential landscape has a time dependent diffusion constant which means that its average mean squared displacement (MSD) only becomes linear at late times. The long time, or effective diffusion constant, can be estimated from the slope of a linear fit of the MSD at late times. Due to the cross over between a short time microscopic diffusion constant, which is independent of the potential, to the effective late time diffusion constant, a linear fit of the MSD will not in general pass through the origin and will have a non-zero constant term. Here we address how to compute the constant term and provide explicit results for Brownian particles in one dimension in periodic potentials. We show that the constant is always positive and that at low temperatures it depends on the curvature of the minimum of the potential. For comparison we also consider the same question for the simpler problem of a symmetric continuous time random walk in discrete space. Here the constant can be positive or negative and can be used to determine the variance of the hopping time distribution.
\end{abstract}

PACS numbers: 05.10.Gg, 02.50.Ey

\section{INTRODUCTION}

Brownian motion is one of the most ubiquitous transport phenomena in Nature, thermally induced molecular collisions in a solvent impart momentum to small particles and they hence acquire a random velocity [1]. In over damped systems, where inertia is overcome by viscous damping, this velocity becomes rapidly uncorrelated in time. In this case the particle's velocity becomes an effective white noise and its position is what is mathematically known as pure Brownian motion. The diffusion equation describing the probability density (PDF) of pure Brownian motion in an isotropic medium in $d$ dimensions is

$$
\frac{\partial p(\mathbf{x}, t)}{\partial t}=\kappa \nabla^{2} p(\mathbf{x}, t)
$$

The term $\kappa$ above is the short time or microscopic diffusion constant. A key quantity which can be extracted from single particle tracking is the mean squared displacement (MSD) whose average is given by

$$
\left\langle\left(\mathbf{X}_{t}-\mathbf{X}_{0}\right)^{2}\right\rangle=2 d \kappa(t) t .
$$

Here $\kappa(t)$ is the effective time dependent diffusion constant. The late time or effective diffusion constant is defined via the limit

$$
\kappa_{e}=\lim _{t \rightarrow \infty} \kappa(t) .
$$

In the case of pure Brownian motion we have $\kappa(t)=\kappa=\kappa_{e}$.

In Nature Brownian particles also interact with their environment via long range interactions that can be generated by electrostatic and other interactions. As well as the noise leading to Brownian motion, the particle also feels a force, or drift, due to this potential. For instant a particle diffusing in a cellular cytoplasm will locally diffuse, but also interact via steric interactions with the cells organelles. The local or short time diffusion constant is solely determined by the nature of the solvent, however at late times the diffusion constant will be modified due to the potential felt by the particle. In the presence of drift due to an external force it is easy to see that $\kappa(0)=\kappa$, i.e. the small time limit of the effective diffusion constant is given by the microscopic diffusion constant. However at late times the diffusion constant will be modified by the potential acting on the particle. The value of $\kappa_{e}$ can be computed exactly in one dimension for any potential [2]. However, except for a particular case in two dimensions [3], in higher dimensions no results are known. The problem of diffusion in a random potential has been extensively studied, in the cases where the diffusion constant exists one can use field theoretic methods such as the perturbation theory and the renormalization group to estimate it for both Gaussian [4, 5] and non-Gaussian potentials [3, 6, 8]. A review of these results can be found in 9 . More recently, due to increasing interest in the physics of non-equilibrium steady states, there have been 
many studies of diffusion in tilted periodic potentials, which are periodic potentials plus a constant linear potential leading to an component with a constant applied force [10.

The presence of a potential acting upon a Brownian particle will cause the effective trajectory to be non-pure Brownian and non-Gaussian. The effective diffusion constant must switch over from the microscopic one to the effective one, meaning that $\kappa(t)$ will vary with time. It can be shown that $\kappa_{e}<\kappa$, essentially due to trapping in the local minima of the potential, so $\kappa(t)$ must decrease with time toward its asymptotic value. Consequently the MSD is concave rather than linear as in the case of Brownian motion, for instance see Fig (1) which is for particles diffusing in a cosine potential. The concave form of the MSD is superficially reminiscent of the sub-diffusive behavior $\left\langle(\mathbf{X}(t)-\mathbf{X}(0))^{2}\right\rangle \sim t^{\alpha}$ where $\alpha<1$ [1]. It is thus conceivable that a slow crossover from the microscopic diffusion constant to the effective one could be misconstrued as anomalous sub-diffusion if the trajectories are not observed over a time sufficient to attain the asymptotic linear diffusive regime of the MSD. The nature of the cross over is thus important to understand, both for discerning between anomalous and ordinary diffusion and because the crossover may contain information about the potential that the particle is subjected to. In this paper we will address the question of how an ultimately diffusive system attains the diffusive regime.

To start with, we consider the finite time correction for continuous time random walks (CTRW). The finite time corrections here are quite easy to compute but the results are nonetheless illuminating and somewhat surprising. Furthermore, at a coarse grained level a Brownian particle diffusing in a periodic potential can be viewed as a CTRW. We then use a Kubo like formula, first given in [6], to analyze finite time corrections for diffusion in a periodic potential and give explicit results for the case of one dimension. Our results are also verified with numerical simulations based on the direct integration of the Langevin equation for an ensemble of tracer particles.

\section{CONTINUOUS TIME RANDOM WALKS}

To start with we consider the simple problem of CTRW in one dimensions on the set of integers. The waiting time at each site has the same distribution $p(\tau)$. After waiting for time $\tau_{i}$ at site $i$ the process hops to the left or right with probability $1 / 2$. In terms of the number of jumps $N(t)$ taken up till time $t$ the mean squared displacement is just given by

$$
\left\langle X_{t}^{2}\right\rangle=N(t),
$$

where $N(t)$ is itself a random variable depending on the waiting times, and the average $\langle\cdot\rangle$ is over the random variables corresponding to a jump to the left or right. The full average is given by

$$
\overline{\left\langle X_{t}^{2}\right\rangle}=\overline{N(t)}
$$

where the average $:$ indicates the average with respect to the waiting times. Now consider an exponentially distributed random time $T$ with rate $s$. Consider the random variable $N(T)$, the number of jumps made before the time $T$ occurs. The probability that a single jump occurs before time $T$ is given by $p_{1}=\operatorname{Prob}(T>\tau)$ which is given by

$$
p_{1}=\int_{0}^{\infty} d \tau \int_{0}^{\infty} d \tau^{\prime} s \exp \left(-s \tau^{\prime}\right) p(\tau) \theta\left(\tau^{\prime}-\tau\right)=\int_{0}^{\infty} d \tau p(\tau) \exp (-s \tau)=\tilde{p}(s),
$$

were $\theta$ is the heaviside function and $\tilde{p}(s)$ is the Laplace transform of $p(\tau)$. Now using the memoryless property of the exponential distribution we see that the probability that there are $n$ steps taken before $T$ is given by

$$
P(n)=\tilde{p}(s)^{n}(1-\tilde{p}(s)),
$$

from which we find that

$$
\mathbb{E}(\bar{N}(T))=\frac{\tilde{p}(s)}{1-\tilde{p}(s)},
$$

where $\mathbb{E}(\cdot)$ denotes the average over $T$. Recalling that the probability distribution function of $T$ is given by $\rho(t)=$ $s \exp (-s t)$ means that Eq. (8) can be written as

$$
\widetilde{\widetilde{N}}(s)=\frac{1}{s} \frac{\tilde{p}(s)}{1-\tilde{p}(s)} .
$$

Therefore

$$
\overline{\left\langle X_{t}^{2}\right\rangle}=\mathcal{L}^{-1}\left\{\frac{1}{s} \frac{\tilde{p}(s)}{1-\tilde{p}(s)}\right\}
$$


where $\mathcal{L}^{-1}\{\cdot\}$ indicates the inverse Laplace transform with respect to parameter $s$.

The late time behavior of the MSD can be extracted from the small $s$ behavior of the Laplace transform, where we can use

$$
\tilde{p}(s)=\overline{\exp (-s \tau)} \approx 1-s \bar{\tau}+\frac{s^{2}}{2} \bar{\tau}^{2}
$$

in the case where the two first moments of the distribution for $\tau$ exist. This gives

$$
\overline{\left\langle X_{t}^{2}\right\rangle}=\mathcal{L}^{-1}\left\{\frac{1}{s^{2} \bar{\tau}}+\frac{1}{s}\left[\frac{\overline{\tau^{2}}-2 \bar{\tau}^{2}}{2 s \bar{\tau}^{2}}\right]\right\} .
$$

Now inverting the Laplace transform gives

$$
\overline{\left\langle X_{t}^{2}\right\rangle} \approx \frac{t}{\bar{\tau}}+\left[\frac{\overline{\tau^{2}}-2 \bar{\tau}^{2}}{2 \bar{\tau}^{2}}\right]
$$

This means that asymptotically the MSD is simply a straight line, measuring the slope gives the value of $2 \kappa_{e}$ while the intercept with the $y$ axis gives $2 C$. The MSD is usually determined from a finite ensemble of trajectories and even from a single trajectory by slicing up the trajectory and using different points for the origin of the process. The resulting experimental curve can be fitted, for example using a least squares linear fit.

Immediately we see that the effective diffusion constant is given by

$$
\kappa_{e}=\frac{1}{2 \bar{\tau}}
$$

which is intuitively obvious to understand, and shows the necessity of a finite average of $\tau$ to see diffusive behavior. An interesting point to make here is that in experiments one may not necessarily be able to obtain the temporal resolution necessary to see individual jumps and thus measure the average trapping time $\bar{\tau}$, however this can be obtained from the diffusion constant via Eq. (14).

The time dependent diffusion constant $\kappa(t)$ therefore behaves at late times as

$$
\kappa(t)=\kappa_{e}+\frac{1}{t}\left[\frac{\overline{\tau^{2}}-2 \bar{\tau}^{2}}{4 \bar{\tau}^{2}}\right] .
$$

The late time correction to the diffusion constant can be positive or negative depending on the sign of

$$
C=\frac{\overline{\tau^{2}}-2 \bar{\tau}^{2}}{4 \bar{\tau}^{2}}
$$

Usually in fitting procedures to estimate $\kappa_{e}$ the value of the constant $C$ is ignored. Here we see that it contains information about the variance of trapping times. Using the estimate for $\bar{\tau}$ from the fit of $\kappa_{e}$ enables one to obtain and estimate for the variance $\operatorname{var}(\tau)$ from the estimate of $C$.

Despite the simplicity of the above calculation, there are a number of interesting features which emerge. In the case where $\tau$ has an exponential distribution $p(\tau)=\mu \exp (-\mu \tau)$ we find that $C=0$. However in this case we can find the full temporal dependence as

$$
\tilde{p}(s)=\frac{\mu}{\mu+s}
$$

which yields

$$
\overline{\left\langle X_{t}^{2}\right\rangle}=\mathcal{L}^{-1}\left\{\frac{\mu}{s^{2}}\right\}=\mu t
$$

i.e. the asymptotic diffusive regime sets in immediately.

We also note that the term $C$ can be written as

$$
C=\frac{\operatorname{var}(\tau)-\bar{\tau}^{2}}{4 \bar{\tau}^{2}}=\frac{1}{4}\left(c_{v}^{2}-1\right),
$$


where $\operatorname{var}(\tau)$ is the variance and $c_{v}=\sqrt{\operatorname{var}(\tau)} / \bar{\tau}$ is the corresponding coefficient of variation of the parental waitingtime distribution. Therefore if $\tau$ has a large variance (so that $c_{v}>1$ ) the late time correction to $\kappa(t)$ tends to be positive, however if the distribution is highly peaked (and $c_{v}<1$ ) then the correction will be negative. The finite time correction for peaked distributions is easy to understand by considering the deterministic hopping case where $p(\tau)=\delta\left(\tau-\tau_{0}\right)$ up to an infinitesimal dispersion of the distribution about $\tau_{0}$. Working in terms of the average jump number we find that

$$
\overline{N(t)}=\frac{t}{\tau_{0}}-\frac{1}{2}
$$

However we know that

$$
N(t)=\frac{t}{\tau_{0}}-R\left(\frac{t}{\tau_{0}}\right)
$$

where $R(u)$ is the non-integer part of $u$. The temporal average value of of this term is given by,

$$
\overline{R\left(\frac{t}{\tau_{0}}\right)}=\int_{0}^{1} d u u=\frac{1}{2}
$$

which trivially explains the result for very peaked distributions, the constant $C$ is essentially negative due to the rounding effect of a discrete random walk.

Another distribution which is interesting to analyze is the power law distribution given by

$$
p(\tau)=\frac{\alpha \tau_{0}^{\alpha}}{\tau^{1+\alpha}}
$$

with $\tau_{0}$ a cut-off timescale below which $p(\tau)=0$. The above analysis above goes through when $\overline{\tau^{2}}$ is finite, that is when $\alpha>2$. In this case we find

$$
C=\frac{-\alpha^{2}+2 \alpha+1}{4 \alpha(\alpha-2)}=-\frac{(\alpha-\sqrt{2}-1)(\alpha+\sqrt{2}-1)}{4 \alpha(\alpha-2)} .
$$

Therefore there is a window of values of $\alpha$ such that $2<\alpha<\sqrt{2}+1$ such that $C>0$, and for all other values of $\alpha>\sqrt{2}+1$ we have $C<0$.

Now consider the case where $\alpha \in(1,2)$ that is to say the mean exists but the variance diverges. In this case we find that

$$
\tilde{p}(s)=1-s \bar{\tau}+s^{\alpha} \tau_{0}^{\alpha} \alpha \int_{s \tau_{0}}^{\infty} \frac{\exp (-u)-1+u}{u^{\alpha+1}} .
$$

Now in the limit where $s \rightarrow 0$ we can approximate the above by

$$
\tilde{p}(s)=1-s \bar{\tau}+s^{\alpha} \tau_{0}^{\alpha} \alpha \int_{0}^{\infty} \frac{\exp (-u)-1+u}{u^{\alpha+1}},
$$

as the integral on the left hand side converges around $u=0$ for $\alpha<2$. This gives, for late times,

$$
\overline{\left\langle X_{t}^{2}\right\rangle} \approx \mathcal{L}^{-1}\left\{\frac{1}{s^{2} \bar{\tau}}+\frac{\alpha H(\alpha) s^{\alpha-3} \tau_{0}^{\alpha}}{\bar{\tau}^{2}}\right\}=\frac{t}{\bar{\tau}}+\frac{\alpha H(\alpha) \tau_{0}^{\alpha} t^{2-\alpha}}{\bar{\tau}^{2} \Gamma(3-\alpha)}
$$

where

$$
H(\alpha)=\int_{0}^{\infty} d u \frac{\exp (-u)-1+u}{u^{\alpha+1}}=\Gamma(-\alpha) .
$$

This can then be simplified, using the factorial property of the Gamma function $\Gamma(z)=(z-1) \Gamma(z-1)$, to obtain

$$
\overline{\left\langle X_{t}^{2}\right\rangle} \approx \mathcal{L}^{-1}\left\{\frac{1}{s^{2} \bar{\tau}}+\frac{\alpha H(\alpha) s^{\alpha-3} \tau_{0}^{\alpha}}{\bar{\tau}^{2}}\right\}=\frac{t}{\bar{\tau}}+\frac{\tau_{0}^{\alpha} t^{2-\alpha}}{(\alpha-1)(2-\alpha) \bar{\tau}^{2}}
$$

We see that as the diffusion tends towards the point where it becomes anomalous (sub-diffusive), the finite time corrections become more important. This is thus an example of a censorship phenomenon for the transition between 
diffusive and sub-diffusive transport. As the transition is approached the finite time corrections to the MSD decay more and more slowly and become of the order of the leading, diffusive, term. The effective diffusion constant close to the transition point thus becomes impossible to measure if one does not know how to include finite time corrections in the fitting procedure used to extract $\kappa_{e}$. A concrete example of this was demonstrated for the case of a particle diffusing in a potential given by the square of a Gaussian function [7], a system which exhibits a transition from a normal to anormal diffusive regime.

Consider now the case $\alpha=\sqrt{2}+1$, where $C$ in Eq. $\sqrt{19}$ is equal to zero, and address the question of the thermalization dynamics for this critical value of $\alpha$. For $2<\alpha<3$, i.e. when the second moment of the waiting time distribution in Eq. 23 exists, while the third one does not, we have

$$
\tilde{p}(s) \approx 1-\bar{\tau} s+\frac{\overline{\tau^{2}}}{2} s^{2}-\alpha \tau_{0}^{\alpha} h(\alpha) s^{\alpha}
$$

where

$$
h(\alpha)=-\int_{0}^{\infty} \frac{d u}{u^{\alpha+1}}\left(e^{-u}-1+u-\frac{u^{2}}{2}\right)=-\Gamma(-\alpha) .
$$

Consequently, we have that for $2<\alpha<3$,

$$
\left\langle\overline{X_{t}^{2}}\right\rangle \approx L^{-1}\left\{\frac{1}{\bar{\tau} s^{2}}\left(1+\left(\frac{\overline{\tau^{2}}}{2 \bar{\tau}}-\bar{\tau}\right) s-\frac{\alpha \tau_{0}^{\alpha} h(\alpha)}{\bar{\tau}} s^{\alpha-1}\right)\right\}
$$

Upon inverting the Laplace transform we find that

$$
\left\langle\overline{X_{t}^{2}}\right\rangle \approx \frac{t}{\bar{\tau}}+\left[\frac{\overline{\tau^{2}}-2 \bar{\tau}^{2}}{2 \bar{\tau}^{2}}\right]-\frac{\tau_{0}^{\alpha} t^{2-\alpha}}{\bar{\tau}^{2}(\alpha-1)(\alpha-2)}
$$

The latter equation implies that in the critical case $\alpha=\sqrt{2}+1$ the long-time relaxation proceeds as

$$
\kappa(t) \approx \frac{1}{\sqrt{2}(\sqrt{2}+1) \tau_{0}}\left(1-\frac{\tau_{0}^{\sqrt{2}}}{t^{\sqrt{2}}}\right)
$$

i.e., the diffusion coefficient approaches its equilibrium value from below and as a power-law with the exponent $\sqrt{2}$. We note that a similar singular behavior - i.e., the change of the dynamical exponent characteristic of the thermalization kinetics - is not specific to the power-law distribution in Eq. 23 but may show up for any underlying waiting-time distribution $p(\tau)$ in which, by tuning some parameters, one manages to tune the coefficient of $\operatorname{variation} \sqrt{\operatorname{var}(\tau)} / \bar{\tau}$ of the underlying distribution $p(\tau)$ to be equal to 1 .

\section{BROWNIAN PARTICLES IN PERIODIC POTENTIALS}

Now having considered the finite time correction for continuous time random walks on discrete space, where we have seen that the spatial discreteness can lead to a trivial rounding type error, let us consider a Langevin process diffusing in a periodic potential.

Here we examine a locally Brownian particle whose probability density function (PDF) obeys the Fokker-Planck equation

$$
\frac{\partial}{\partial t} p(\mathbf{x}, t)=\kappa \nabla \cdot(\nabla p(\mathbf{x}, t)+\beta p(\mathbf{x}, t) \nabla \phi(\mathbf{x}))=-H p(\mathbf{x}, t) .
$$

Here $\phi$ is a potential which is periodic and finite, so the diffusion constant at late times exists. In any dimension the late time effective diffusion constant is given by [6, 13 ]

$$
\kappa_{e}=\kappa-\frac{\kappa^{2} \beta^{2}}{d} \int d \mathbf{x} d \mathbf{x}_{0} \nabla \phi(\mathbf{x}) \cdot H^{-1}\left(\mathbf{x}, \mathbf{x}_{0}\right) \nabla \phi\left(\mathbf{x}_{0}\right) p_{e q}\left(\mathbf{x}_{0}\right)
$$

This result can be demonstrated by adapting a method introduced by Derrida 12 for discrete random walks to the case of Langevin processes [13] or by direct manipulation of the Langevin equation [6] to obtain a Kubo-like formula 
for the late time diffusion constant. However, if we consider a periodic potential in a region of space much larger than the period length and assume that the particle is in equilibrium (specifically we mean that the position of the particle modulo the period is in equilibrium), it can be shown, directly from the Langevin equation [6], that

$$
\kappa(t)=\kappa_{e}+\frac{\kappa^{2} \beta^{2}}{d t} \int d \mathbf{x} d \mathbf{x}_{0} \nabla \phi(\mathbf{x})\left[H^{-2}(1-\exp (-t H))\right]\left(\mathbf{x}, \mathbf{x}_{0}\right) \cdot \nabla \phi\left(\mathbf{x}_{0}\right) p_{e q}\left(\mathbf{x}_{0}\right) .
$$

In the above, $p_{e q}(\mathbf{x})$ is the equilibrium probability distribution over a region of large but finite size $L$.

To start our analysis of these formulas we start by considering the correction to the late time, effective, diffusion constant by the presence of a potential in one dimension. We write $\kappa_{e}=\kappa-\Delta \kappa$ where

$$
\Delta \kappa=\kappa^{2} \beta^{2} \int d x d x_{0} \frac{d \phi(x)}{d x} H^{-1}\left(x, x_{0}\right) \frac{d \phi\left(x_{0}\right)}{d x_{0}} p_{e q}\left(x_{0}\right),
$$

and where the equilibrium distribution over the interval $[0, L]$ is given by

$$
p_{e q}(x)=\frac{\exp (-\beta \phi(x))}{L\langle\exp (-\beta \phi)\rangle}
$$

In the above we have defined the spatial average of the Boltzmann weight

$$
\langle\exp (-\beta \phi)\rangle=\frac{1}{L} \int_{0}^{L} d x \exp (-\beta \phi(x)) .
$$

Now we define

$$
f(x)=\int d x_{0} H^{-1}\left(x, x_{0}\right) \frac{d \phi\left(x_{0}\right)}{d x_{0}} p_{e q}\left(x_{0}\right),
$$

and thus $f$ obeys

$$
H f=-\kappa \frac{d}{d x}\left(\frac{d f}{d x}+\beta \frac{d \phi}{d x} f\right)=\frac{d \phi(x)}{d x} p_{e q}\left(x_{0}\right) .
$$

This second order differential equation can be integrated to give

$$
f(x)=\frac{1}{\kappa \beta L\langle\exp (-\beta \phi)\rangle} \exp (-\beta \phi(x))\left[x+c \int_{0}^{x} d x^{\prime} \exp \left(\beta \phi\left(x^{\prime}\right)\right)+b\right],
$$

where $c$ and $b$ are two integration constants that must be determined. To determine the constants we now consider a periodic potential with period $l$ such that $l \ll L$. The function $f$ must therefore be periodic with the same period, and this gives

$$
c=-\frac{l}{\int_{x}^{x+l} d x^{\prime} \exp \left(\beta \phi\left(x^{\prime}\right)\right)}=-\frac{1}{\langle\exp (\beta \phi)\rangle} .
$$

At this point we do no have to identify the constant $b$ as we have

$$
\Delta \kappa=\kappa^{2} \beta^{2} \int d x \frac{d \phi(x)}{d x} f(x),
$$

and the term proportional to $b$ in $f(x)$ will contribute 0 to $\Delta \kappa$ due to the periodicity of the potential. Performing the integrals we find

$$
\Delta \kappa=\frac{\kappa^{2} \beta^{2}}{\kappa \beta L\langle\exp (-\beta \phi)\rangle} \frac{L}{\beta}\left(\langle\exp (-\beta \phi)\rangle-\frac{1}{\langle\exp (\beta \phi)\rangle}\right),
$$

which simplifies to give

$$
\Delta \kappa=\kappa-\frac{\kappa}{\langle\exp (\beta \phi)\rangle\langle\exp (-\beta \phi)\rangle},
$$


and, eventually,

$$
\kappa_{e}=\frac{\kappa}{\langle\exp (\beta \phi)\rangle\langle\exp (-\beta \phi)\rangle}
$$

This is a standard result on one dimensional diffusion which has been known for a long time and has been derived with a wide variety of different methods [2. Interestingly the derivation we present here is based on the direct evaluation of the MSD, whereas in all the other derivations we are aware of [2] a mean first passage time argument is used and then turned around to give the diffusion constant.

We now consider the finite time corrections. Our results on CTRWs suggest that the first term in the finite time integral Eq. (37) will be the leading correction, i.e.

$$
\kappa(t) \approx \kappa_{e}+\frac{C}{t}
$$

where

$$
C=\frac{\kappa^{2} \beta^{2}}{d} \int d \mathbf{x} d \mathbf{x}_{0} \nabla \phi(\mathbf{x})\left[H^{-2}\right]\left(\mathbf{x}, \mathbf{x}_{0}\right) \cdot \nabla \phi\left(\mathbf{x}_{0}\right) p_{e q}\left(\mathbf{x}_{0}\right)
$$

Staying again in one dimension, this can be written as

$$
C=\kappa^{2} \beta^{2} \int d x g(x) f(x)
$$

where $f(x)$ is as defined by Eq. (41) and we have used the fact that $H^{-1 \dagger}=H^{\dagger-1}$ (where $\dagger$ denotes the adjoint) to introduce

$$
g(x)=\int d x_{0} H^{\dagger-1}\left(x, x_{0}\right) \frac{d \phi\left(x_{0}\right)}{d x_{0}} .
$$

This means that rather than solve a fourth order differential equation, we need only solve two second order ones. The first equation for $f$ is already solved in Eq. (43) and the equation for $g$ is given by

$$
-\kappa\left(\frac{d^{2} g}{d x^{2}}-\beta \frac{d \phi}{d x} \frac{d g}{d x}\right)=\frac{d \phi}{d x}
$$

This equation integrates to give

$$
g(x)=\frac{1}{\kappa \beta}\left[x-\frac{1}{\langle\exp (\beta \phi)\rangle} \int_{0}^{x} d x^{\prime} \exp \left(\beta \phi\left(x^{\prime}\right)\right)+b^{\prime}\right],
$$

where the periodicity of $g$ has again been used to determine one of the constants of integration.

We thus see that the two functions $f$ and $g$ are related and we can write

$$
\begin{aligned}
f(x) & =\frac{1}{\kappa \beta L\langle\exp (-\beta \phi)\rangle} \exp \left(-\beta \phi\left(x^{\prime}\right)\right)[R(x)+b] \\
g(x) & =\frac{1}{\kappa \beta}\left[R(x)+b^{\prime}\right]
\end{aligned}
$$

where

$$
R(x)=L\left(\frac{x}{L}-\frac{1}{L\langle\exp (\beta \phi)\rangle} \int_{0}^{x} d x^{\prime} \exp \left(\beta \phi\left(x^{\prime}\right)\right)\right) .
$$

Before we proceed, it is interesting to note that $R(x)$ in Eq. (56) has a probabilistic interpretation. The first term in the brackets on the right-hand-side of Eq. (56) defines the so-called hitting probability in absence of an external potential (see, e.g. [17]) - the probability that a free diffusion on the interval [0,L], starting at $x$, will first hit the point $x=L$ without ever hitting the left extremity of the interval. In turn, the second term on the right-hand-side of Eq. (56) is exactly the analogous hitting probability for diffusion in presence of the potential $-\phi(x)$ (see, e.g. [18). 
The problem we are now faced with is that of determining the constants $b$ and $b^{\prime}$. First let us consider the general solution to an equation of the form Eq. (41) for the function $f(x)$. It can be rewritten up to a constant multiplicative factor, which we drop for notational convenience, as

$$
f(x)=\int_{0}^{\infty} d t \int d x_{0}[\exp (-t H)]\left(x, x_{0}\right) \frac{d \phi\left(x_{0}\right)}{d x_{0}} \exp \left(-\beta \phi\left(x_{0}\right)\right) .
$$

The integral over $t$ will converge as the function $\frac{d \phi\left(x_{0}\right)}{d x_{0}} \exp \left(-\beta \phi\left(x_{0}\right)\right)$ is orthogonal to the left eigenfunction (which is constant) of eigenvalue 0 of the Fokker-Planck operator $H$. However we have

$$
[\exp (-t H)]\left(x, x_{0}\right)=p\left(x, x_{0}, t\right)
$$

where $p$ is the transition density for the process. Conservation of probability $\int d x p\left(x, x_{0}, t\right)=1$ then gives

$$
\int d x f(x)=\int_{0}^{\infty} d t \int d x_{0} \int d x p\left(x, x_{0}, t\right) \frac{d \phi\left(x_{0}\right)}{d x_{0}} \exp \left(-\beta \phi\left(x_{0}\right)\right)=\int_{0}^{\infty} d t \int d x_{0} \frac{d \phi\left(x_{0}\right)}{d x_{0}} \exp \left(-\beta \phi\left(x_{0}\right)\right)=0
$$

where we have used periodic boundary conditions. This means that the constant $b^{\prime}$ associated with the function $g$ does not contribute to the calculation and we have

$$
C=\frac{1}{L\langle\exp (-\beta \phi)\rangle} \int d x \exp (-\beta \phi(x))\left[R^{2}(x)+b R(x)\right]
$$

where $b$ is determined by $\int d x f(x)=0$ and thus

$$
b=-\frac{\int d x \exp (-\beta \phi(x)) R(x)}{L\langle\exp (-\beta \phi)\rangle} .
$$

Now if we denote averages with respect to the equilibrium Gibbs-Boltzmann measure via

$$
\langle A(x)\rangle_{e q}=\frac{\int_{0}^{L} d x \exp (-\beta \phi(x)) A(x)}{L\langle\exp (-\beta \phi)\rangle},
$$

we can write

$$
C=\left\langle R^{2}(x)\right\rangle_{e q}-\langle R(x)\rangle_{e q}^{2}
$$

Note also that due to the periodicity of $\phi$ and $R$ with respect to $l$ the above averages can be computed over an interval of length $l$. An interesting consequence of this result is that the value of $C$ is always positive, in contrast to the CTRW case. Another interesting feature of this formula for $C$ is that it is independent of $\kappa$.

Now consider the case where the potential is given by

$$
\phi(x)=V\left(\frac{2 \pi x}{l}\right)
$$

where $V$ has period $2 \pi$ giving $\phi$ a period $l$. Making the change of variables $z=2 \pi x / l$ in the integral expressions for $C$ we have

$$
\left\langle R^{2}(x)\right\rangle_{e q}=\frac{l^{2}}{(2 \pi)^{2} \int_{0}^{2 \pi} d z^{\prime} \exp \left(-\beta V\left(z^{\prime}\right)\right)} \int_{0}^{2 \pi} d z \exp (-\beta V(z))\left[z-\frac{\int_{0}^{z} d z^{\prime} \exp \left(\beta V\left(z^{\prime}\right)\right)}{\frac{1}{2 \pi} \int_{0}^{2 \pi} d z^{\prime} \exp \left(\beta V\left(z^{\prime}\right)\right)}\right]^{2} .
$$

and

$$
\langle R(x)\rangle_{e q}=\frac{l}{(2 \pi) \int_{0}^{2 \pi} d z^{\prime} \exp \left(-\beta V\left(z^{\prime}\right)\right)} \int_{0}^{2 \pi} d z \exp (-\beta V(z))\left[z-\frac{\int_{0}^{z} d z^{\prime} \exp \left(\beta V\left(z^{\prime}\right)\right)}{\frac{1}{2 \pi} \int_{0}^{2 \pi} d z^{\prime} \exp \left(\beta V\left(z^{\prime}\right)\right)}\right]
$$

This means that $C=c l^{2}$, where $c$ is a constant independent of $l$. In the limit $\beta \rightarrow \infty$, for sufficiently smooth potentials, both $\kappa_{e}$ and $C$ can be evaluated the saddle point method. The effective diffusion constant takes the Arrhenius, and more precisely Kramers [14, form

$$
\kappa_{e}=\kappa 2 \pi \beta \sqrt{\left|V^{\prime \prime}\left(z_{\max }\right)\right| V^{\prime \prime}\left(z_{\min }\right)} \exp \left(-\beta\left(V\left(z_{\max }\right)-V\left(z_{\min }\right)\right),\right.
$$




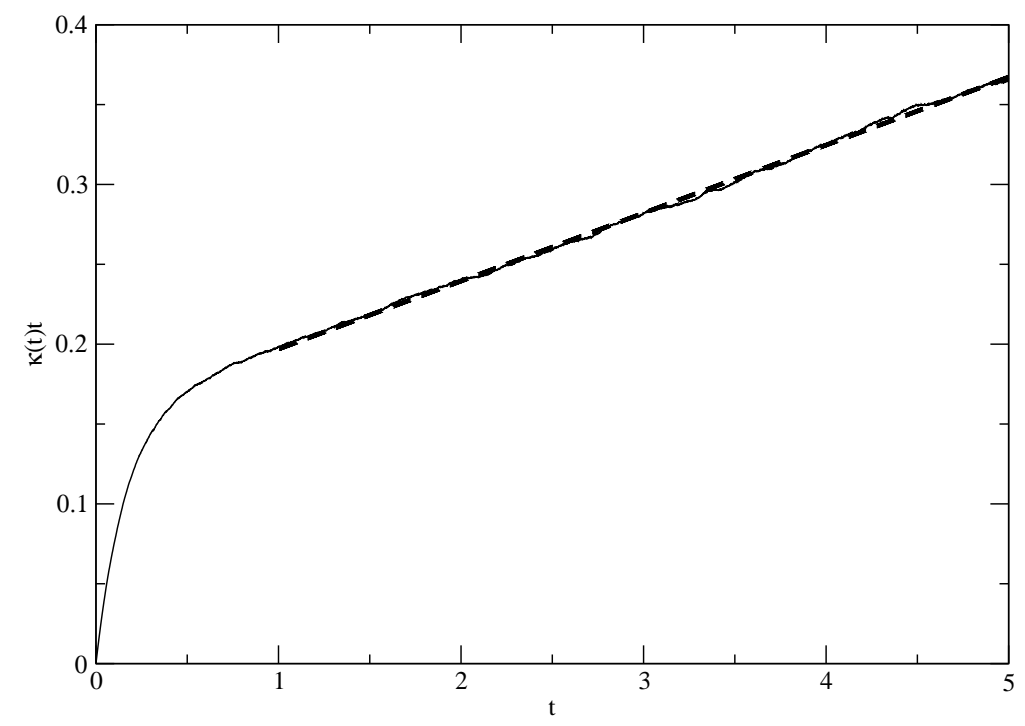

FIG. 1: $\quad \kappa(t) t$ estimated from the MSD in a numerical simulation of $10^{5}$ Langevin particles in the potential in Eq. 64 given by $V(x)=\cos (x), \beta=3$ and $l=4.0$ (continuous) black line. Shown as thick dashed line is the linear fit of $\kappa(t) t$ for $t>1$ yielding the estimate $\kappa_{e}=0.04258$ and $C=0.15432$. The analytical predictions are $\kappa_{e}=0.04198$ and $C=0.15778$.

where $z_{\max }$ and $z_{\min }$ are respectively the points where the potential takes its maximal and minimum values respectively. In the same regime the constant $C$ is given by

$$
C=\frac{l^{2}}{(2 \pi)^{2} \beta V^{\prime \prime}\left(z_{\min }\right)}
$$

We therefore see that the diffusion constant at low temperatures is dominated by an Arrhenius like term involving the maximal energy barrier in the system, while the prefactors depends on the curvature of the maximum and minimum of the potential. The constant term $C$ however only depends on the the curvature of the minimum of the potential.

The predictions can be tested by simulating the Langevin equation. The particles are initially started at the same point and are then allowed to diffuse during an equilibriation time where the variable $x$ modulo the period $l$ can equilibriate. The initial conditions $X(0)$ for each particle used for the computation of the MSD $\left\langle(X(t)-X(0))^{2}\right\rangle=$ $2 \kappa(t) t$ are given by this equilibriation step. Two sources of error are present in the simulation (i) the use of a finite time step $d t$ and also the statistical fluctuations due to using a finite number of particles in the simulations. In the cases where $\kappa_{e}$ and $C$ are sufficiently large, that is to say larger than 0.1 for $\kappa_{e}$ and 0.1 for $C$, the fluctuations (the difference in the fits for $\kappa_{e}$ and $C$ between two distinct simulations for the same number of particles but different random seed for the simulations) are of the order of $5 \%$ of the measured values if we take $10^{5}$ particles. The time step is taken to be $d t=0.0005$ (we checked that the results did not differ beyond the $5 \%$ error in going between $d t=0.001$ and $d t=0.0005$ ). The MSD at each time is computed by ensemble averaging over each individual particle's squared displacement. From the MSD we plot the resulting estimate for $\kappa(t) t$. An example is shown in Fig (1). We see that after a certain time the MSD curve becomes linear, in this case for $t>1$. From the linear fit in this region the resulting estimates for $\kappa_{e}$ and $C$ are extracted and we find that they are in excellent agreement with our analytical predictions.

The explicit calculations carried out here are of course relevant to one dimension. However from the general form of Eq. (37) we see that in any dimension if the constant $C$ exists then by dimensional analysis we must have that $C=c l^{2}$, where $c$ is independent of the periodicity $l$ of the potential. In addition, using the explicit form of $H$, we see that the constant $C$ must be independent of $\kappa$.

Closed form expressions are not available for the constant $C$ for arbitrary potentials, However, exploiting the exact results in Eqs.(63), (65) and (66) for the amplitude $C$ of the relaxation term, we consider a simple, but instructive, case (see Fig.(2)) where the potential $\phi(x)$ is homogeneous, $\phi(x)=0$, for $0 \leq x<2 \pi \xi$ with $0<\xi<1$, and is 

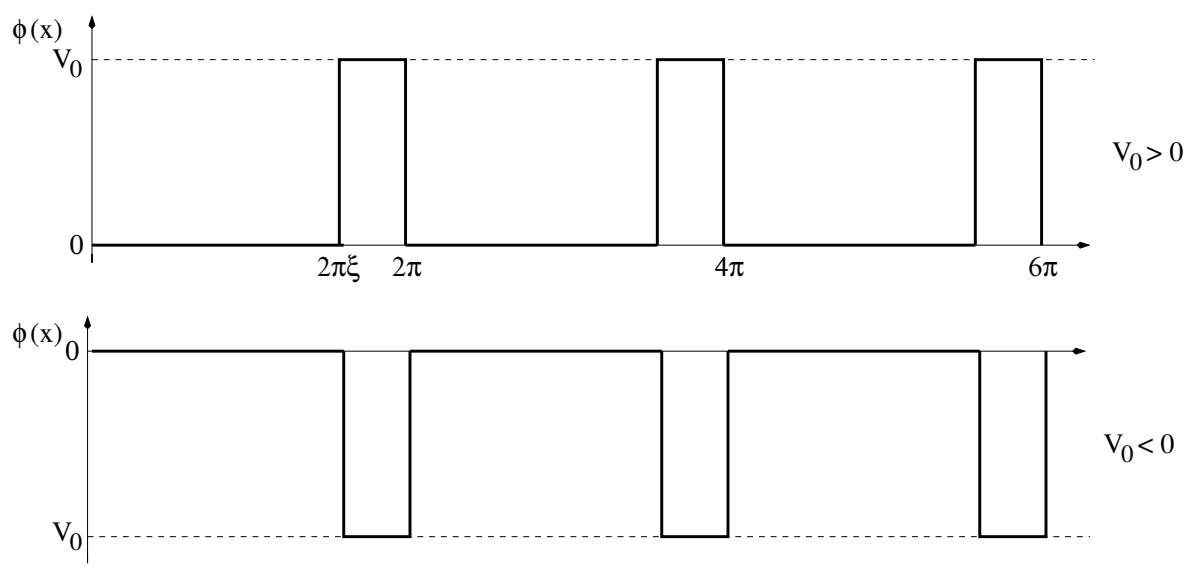

FIG. 2: A homogeneous potential $\phi(x)=0$ perturbed by a narrow barrier with height $V_{0}>0$ or a well of depth $V_{0}<0$.

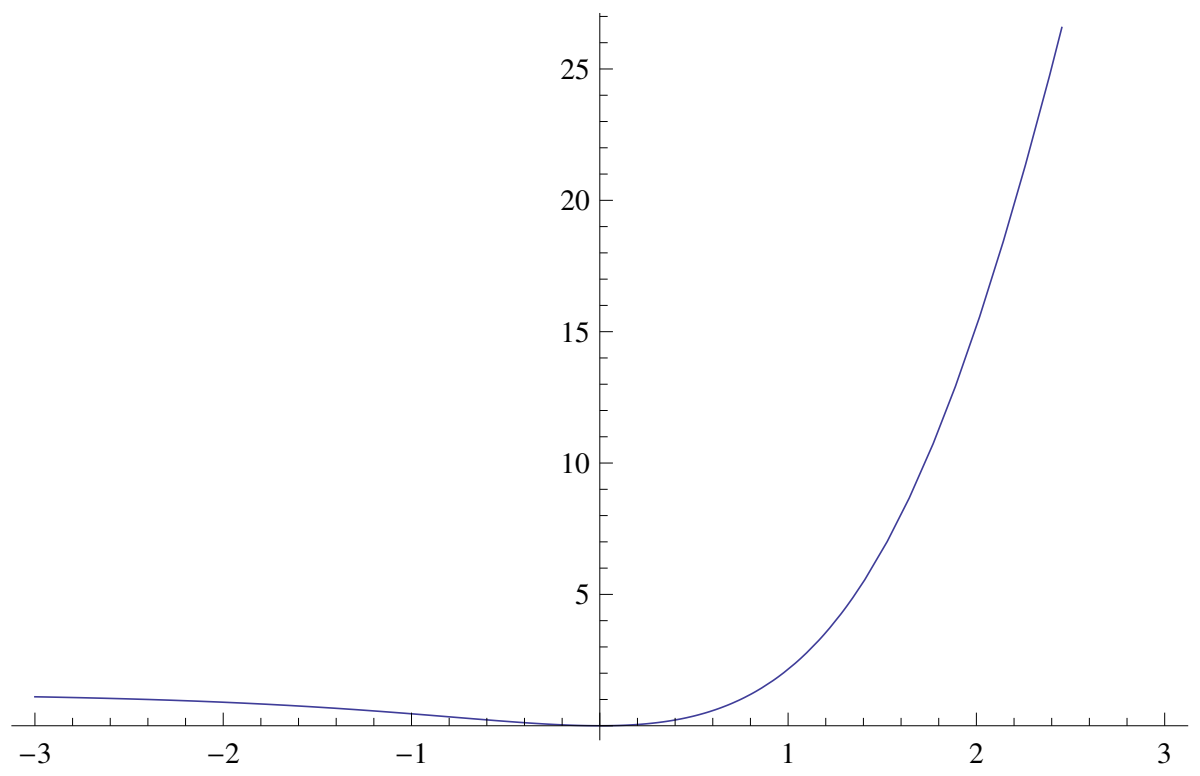

FIG. 3: The scaled relaxation amplitude $12 C / \xi^{2}(1-\xi)^{2} l^{2}$ in $(70)$ versus $\beta V_{0}$ for $\xi=0.9$.

perturbed by a narrow rectangular barrier (well), $\phi(x)=V_{0}$, in the region $2 \pi \xi \leq x \leq 2 \pi$, where $V_{0}>0$ in the case of a barrier and $V_{0}<0$ for a well. For this case it is straightforward to find from Eq. (48) that the diffusion coefficient is given explicitly by

$$
\begin{aligned}
\kappa_{e} & =\frac{\kappa l^{2}}{(2 \pi)^{2}\left(\xi+(1-\xi) \exp \left(\beta V_{0}\right)\right)\left(\left(\xi+(1-\xi) \exp \left(-\beta V_{0}\right)\right)\right.} \\
& =\frac{\kappa l^{2}}{(2 \pi)^{2}\left(\xi^{2}+(1-\xi)^{2}+2 \xi(1-\xi) \cosh \left(\beta V_{0}\right)\right)}
\end{aligned}
$$

Note that $\kappa_{e}$ is an even function of $V_{0}$ which means that the diffusion coefficient $\kappa_{e}$ is the same for both the case of periodic barriers and for the case of the wells with the same, by absolute value, $V_{0}$. Therefore, knowing just the diffusion coefficient $\kappa_{e}$, we are unable to distinguish if the diffusion takes place in presence of barriers or in the presence wells - even if we know $\xi$, upon extracting $\kappa_{e}$ from the numerical data we are only able to infer the absolute value of $V_{0}$ but not its sign. Consider next what information one can extract by studying the amplitude of the relaxation term. From our Eqs. (63), 65) and (66) we obtain

$$
C=\frac{\xi^{2}(1-\xi)^{2} l^{2}}{12}\left(\frac{\exp \left(\beta V_{0}\right)-1}{\xi+(1-\xi) \exp \left(\beta V_{0}\right)}\right)^{2} .
$$


A salient feature of the result in $\sqrt{70}$ is that $C$, in a striking contrast to the diffusion coefficient $\kappa_{e}$, is a strongly asymmetric function of $\beta V_{0}$, as one observes in Fig.(3). Namely, one gets a very different values of $C$ for $V_{0}<0$ and $V_{0}>0$, so that studying the amplitude of the relaxation term we can distinguish between diffusion in presence of barriers or and diffusion in presence of wells.

\section{CONCLUSIONS}

In many experimental situations diffusion constants are determined by a linear fit of an experimentally generated MSD curve. In general Brownian particles are subjected to external forces and this extra drift means that they do not behave as pure Brownian motion. The effect of interaction means that the average MSD only becomes proportional to $t$ at late times. In this region the diffusion constant can be extracted via a fitting procedure, for instance a linear fit (which may not necessarily be the best way to fit such data [15, 16]) will yield a slope proportional to $2 \kappa_{e}$ and also a constant $2 C$ where it intercepts the vertical axis. If the particle is Brownian in an external conservative force field, we have shown that this constant must be positive and in one dimension we have found an analytical expression for it. We believe that these results could be useful in analyzing single particle tracking data, as the constant term, which is usually ignored or not discussed, contains potentially interesting information about the potential landscape seen by the Brownian particle. In particular we have seen that at low temperatures, knowing $C$ enables one to estimate the curvature of the local minima of the potential responsible for slowing diffusion down, while in the Kramers expression for the diffusion constant this information is mixed with the Arrhenius term which contains information about the energy barrier.

A number of questions remain open, here we have examined the leading order correction to the average MSD which turns out to be a constant. The next order corrections would be interesting to analyze as they would allow one to estimate when one achieves the effective linear regime of asymptotic diffusion. Another important question is what happens in the case where the potential is not periodic but say, for instance, random but statistically stationary. In this case the leading order correction is probably time dependent [6].

[1] S. N. Majumdar, Current Science 89, 2076 (2005).

[2] P.G. de Gennes, J. Stat. Phys. 12463 (1975); R. Zwanzig, Proc. Nat. Acad. Sci. 852029 (1988).

[3] D.S. Dean, I.T. Drummond and R.R. Horgan, J. Phys. A 37, 2039 (2004).

[4] D.S. Dean, I.T. Drummond and R.R. Horgan, J. Phys. A 27, 5153 (1994); ibid, J. Phys. A 28, 1235 (1995); ibid, J. Phys. A 28, 6013 (1995); ibid, J. Phys. A. 29, 7867 (1996).

[5] M.W. Deem and D. Chandler, J. Stat. Phys. 76, 911, (1994).

[6] D.S. Dean, I.T. Drummond, R.R. Horgan, A. Lefevre, J. Phys. A.,37, 10459 (2004)

[7] C. Touya and D.S. Dean, J. Phys. A 40 919, (2007).

[8] D.S. Dean and C. Touya, J. Phys. A 41, 335002 (2008).

[9] D.S.Dean, I.T. Drummond and R.R. Horgan, J. Stat. Mech. 7 P07013 (2007).

[10] P. Reimann, C. Van den Broeck, H. Linke, P. Hänggi, J.M. Rubu and A. Pérez-Madrid, Phys. Rev. Lett. 87, 010602 (2001); M. Khoury, A. M. Lacasta, J. M. Sancho and K. Lindenberg Phys. Rev. Lett. 106, 090602 (2011) ; M. S. Simon, J. M. Sancho and K. Lindenberg Phys. Rev. E 88, 062105 (2013).

[11] J.-P. Bouchaud and A. Georges, Phys. Rep. 195, 127 (1990).

[12] B. Derrida, J. Stat. Phys. 31, 433 (1983).

[13] D.S. Dean, I.T. Drummond and R.R. Horgan, J. Phys. A 30, 385 (1997).

[14] H. A. Kramers, Physica 7, 284 (1940).

[15] D. Boyer and D.S. Dean, J. Phys. A: Math. Gen. 44, 335003 (2011);

[16] D. Boyer, D.S. Dean, C. Mejía-Monasterio and G. Oshanin, Phys. Rev. E 85, 031136 (2012); ibid, Phys. Rev. E 86, 060101(R) (2012); ibid, J. Stat. Mech. P04017 (2013).

[17] S. Redner, A Guide to First-Passage Processes (Cambridge University Press), New York, (2001).

[18] G. Oshanin and S. Redner, Eur. Phys. Lett. 85, 10008 (2009). 